\title{
STATIONARY SOLUTIONS OF INCOMPRESSIBLE VISCOUS FLOW IN A WALL-DRIVEN SEMI-CIRCULAR CAVITY
}

\author{
ERCAN ERTURK
}

\begin{abstract}
Istanbul Medeniyet University, Mechanical Engineering Department, Goztepe Campus, 34700 Istanbul, Turkey correspondence: ercan.erturk@medeniyet.edu.tr
\end{abstract}

\begin{abstract}
Stationary numerical solutions of incompressible viscous flow inside a wall-driven semicircular cavity are presented. After a conformal mapping of the geometry, using a body-fitted mesh, the Navier-Stokes equations are solved numerically. The stationary solutions of the flow in a wall-driven semi-circular cavity are computed up to $R e=24000$. The present results are in good agreement with the published results found in the literature. Our results show that as the Reynolds number increases, the sizes of the secondary and tertiary vortices increase, whereas the size of the primary vortex decreases. At large Reynolds numbers, the vorticity at the primary vortex centre increases almost linearly stating that Batchelor's mean-square law is not valid for wall-driven semi-circular cavity flow. Detailed results are presented and also tabulated for future references and benchmark purposes.
\end{abstract}

KEYWORDs: Driven semi-circular cavity flow, large Reynolds number flow, numerical solutions, 2-D incompressible viscous flow.

\section{INTRODUCTION}

Flows in enclosures are studied very frequently in Computational Fluid Dynamics studies since they retain rich flow physics in rather simple geometries. The flow in a lid-driven square cavity is one example for flows in enclosures and the driven cavity flow is studied extensively in the literature and among a very large number of computational studies, the studies [1, [2] and [3] can be given as examples. Other than the flow in a square cavity, in the literature, there are studies on flows in different cavity geometries as well. For example, the flows in a driven skewed cavity $(4,5)$, in a driven triangular cavity $([6]-8])$ and also in a driven trapezoidal cavity ([9]) are studied in numerous numerical studies in the literature. In these enclosures, the formation of the flow structures as the Reynolds number increases attracts the attention of researchers.

Similar to the flows in different enclosures mentioned above, Glowinski et al. [10] studied the flow in a semi-circular cavity. They ([10]) used an unsteady operator-splitting/finite elements method and solved the governing flow equations on an unstructured mesh and presented detailed stationary solutions of the semi-circular cavity flow. Later, Yang et al. [11, 12] and Ding et al. 13 numerically studied the same flow problem using the Lattice Boltzmann method. Also, $\mathrm{Yu}$ et al. 14] solved the Navier-Stokes equations in polar coordinates using a compact difference scheme and simulated the semi-circular cavity flow problem.

Mercan and Atalik [15] considered the arc-shaped cavity flow for the semi-circular case numerically using an unsteady finite difference method. They ([15]) used an elliptic grid generator scheme in order to obtain a body-fitted general coordinates and solve the governing streamfunction and vorticity equations. In their study, they ([15]) have also presented variations of the arc shaped cavity flow with different aspect ratios.

Migeon et al. [16] carried out experiments to study the flow development inside a semi-circular cavity as well as inside square and rectangular cavities. They ([16]) presented qualitative experimental flow visualizations of the semi-circular cavity flow.

At large Reynolds numbers, the stationary flow loses stability and eventually, a transition to turbulence occurs. For the Navier-Stokes equations, the stationary solution still exists at large Reynolds numbers alongside with the transient solutions. As stated in [17] and [18, for the theory of the Navier-Stokes model, it is important to study the stationary solutions at large Reynolds numbers even when the flow loses stability. In order to illustrate the point, the classical flow problem of the incompressible viscous flow around a circular cylinder can be given as an example. For this flow problem, it is well known that the flow is stationary up to Reynolds number of approximately 40. Above this Reynolds number $(R e=40)$, the flow over a circular cylinder becomes unsteady and Karman vortex street appears in the downstream flow field. In the case of vanishing viscosity (i.e. large Reynolds number), Helmholtz-Kirchhoff solution (see [19]) presents a limiting solution when $R e \rightarrow \infty$. In the literature, Smith 20] and Peregrine 21] analysed the laminar incompressible flow past a circular cylinder at large Reynolds numbers mathematically. Also, in the literature, there are numerical studies that analysed the incompressible steady viscous flow past a circular cylinder at large Reynolds numbers (well above $R e=40)$, such as [17, 18, 22,27]. As Christov et al. 27] stated, when $R e \rightarrow \infty$, without dissipation, the limiting solutions of the Navier-Stokes is an important fundamental problem. 
In this study, we numerically investigate the behavior of the stationary solutions of an incompressible viscous flow in a wall-driven semi-circular cavity at large Reynolds numbers. In our study, unlike in [10, 11, 13, 15], as discussed in [28, we use a steady approach and solve the governing steady Navier-Stokes equations. We used complex algebra and mapped the considered semi-circular geometry into an infinite half domain conformally in order to analytically obtain a body-fitted mesh. For the solution of the semicircular cavity flow problem, the streamfunction and vorticity equations are solved iteratively up to large Reynolds numbers. The present numerical results are compared in detail with the results of $10,12,14,15$. For the flows in enclosures, Batchelor's mean square law ([29]) states that the flow, which is coupled to the solid wall velocities at the boundaries, should have a solid body motion inside the enclosure with a uniform vorticity at large Reynolds numbers and the flow quantities should converge to the limiting values. We examine whether or not the Batchelor's model ([29]) is valid for the wall-driven semi-circular cavity flow problem. Detailed results are presented.

\section{NumERICAL METHOD}

The schematic view of the considered flow problem, i.e., the semi-circular cavity flow, is given in Figure 1. The width of the top moving lid, i.e., the straight top side of the semi-circular cavity, is equal to 1 and the velocity of this lid is also equal to 1 .

The steady incompressible viscous flow in a walldriven semi-circular cavity is governed by the NavierStokes equation. We consider the governing equations in streamfunction and vorticity formulation as the following

$$
\begin{gathered}
\psi_{y} \omega_{x}-\psi_{x} \omega_{y}=\frac{1}{R e}\left(\omega_{x x}+\omega_{y y}\right) \\
\psi_{x x}+\psi_{y y}=\omega
\end{gathered}
$$

where $R e$ is the Reynolds number based on the velocity of the top lid $(U)$ and the width of the lid $(L)$ which are both equal to unity.

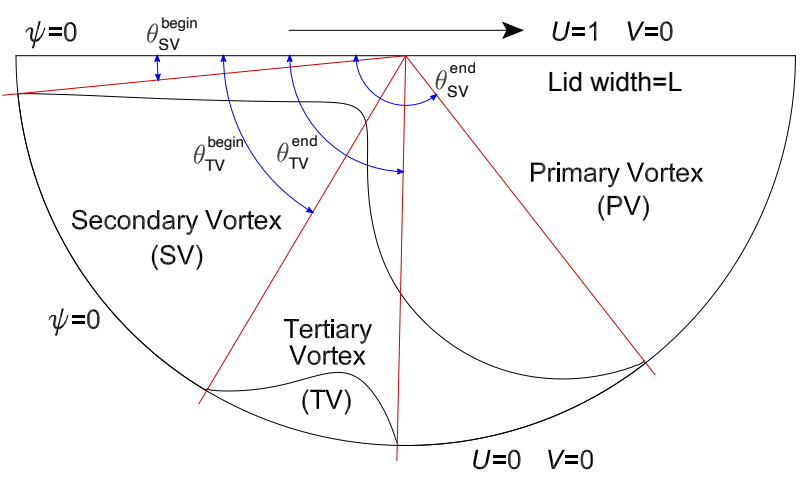

FigURE 1. Schematic view of the considered flow problem, boundary conditions and flow topology of the semi-circular cavity flow.

\subsection{NUMERICAL MESH}

In computational fluid dynamics, it is always preferred to use a body-fitted orthogonal coordinate system for numerical solutions. Using a complex variable analysis, one can obtain that the following complex function

$$
w=\ln \frac{z-\frac{1}{2}}{z+\frac{1}{2}}
$$

conformally maps the considered semi-circular cavity geometry into a body-fitted orthogonal coordinate system. In the mapped complex $w$-domain, the real and imaginary parts are given as $w=\phi+i \psi$ where $\psi$ and $\phi$ are the coordinate axes in the mapped domain. Also, the complex $z$-domain represents the $x$ - $y$ plane as the following $z=x+i y$. We note that, one can obtain this transformation also using the potential flow analysis with the superposition of a source located at $\left(-\frac{1}{2}, 0\right)$ coordinates and a sink located at $\left(\frac{1}{2}, 0\right)$ coordinates with equal strengths of $2 \pi$ as shown in Figure 2

Upon separating the real and imaginary parts, the velocity potential function is the real part where

$$
\phi=\ln \frac{\left|z-\frac{1}{2}\right|}{\left|z+\frac{1}{2}\right|}=\ln \frac{\sqrt{\left(x-\frac{1}{2}\right)^{2}+y^{2}}}{\sqrt{\left(x+\frac{1}{2}\right)^{2}+y^{2}}}
$$

and also the stream function is the imaginary part where

$$
\psi=\arctan \frac{y}{x-\frac{1}{2}}-\arctan \frac{y}{x+\frac{1}{2}}
$$

In this study we used the $\psi$ and $\phi$ coordinates in order to construct a body-fitted mesh for the semicircular cavity. For any value of the velocity potential function, such that $\phi=c$, we have

$$
c=\ln \frac{\sqrt{\left(x-\frac{1}{2}\right)^{2}+y^{2}}}{\sqrt{\left(x+\frac{1}{2}\right)^{2}+y^{2}}}
$$

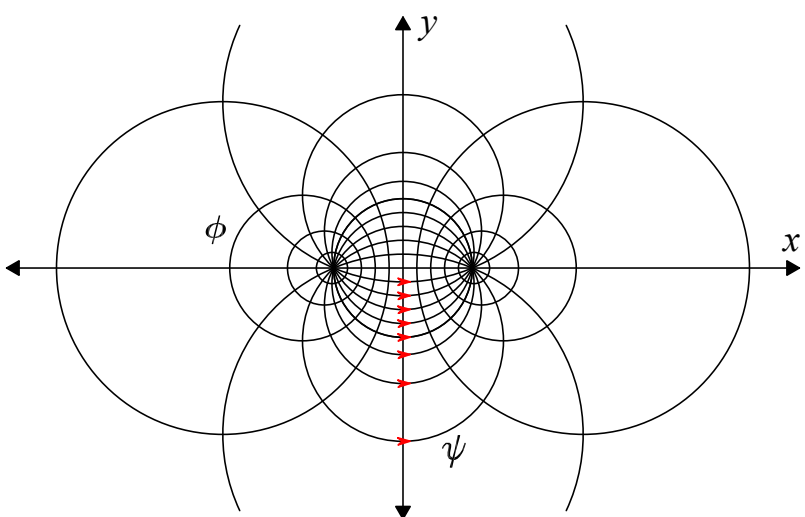

FiguRE 2. Velocity potential and stream function lines of the potential flow with a source and a sink with equal strengths. 


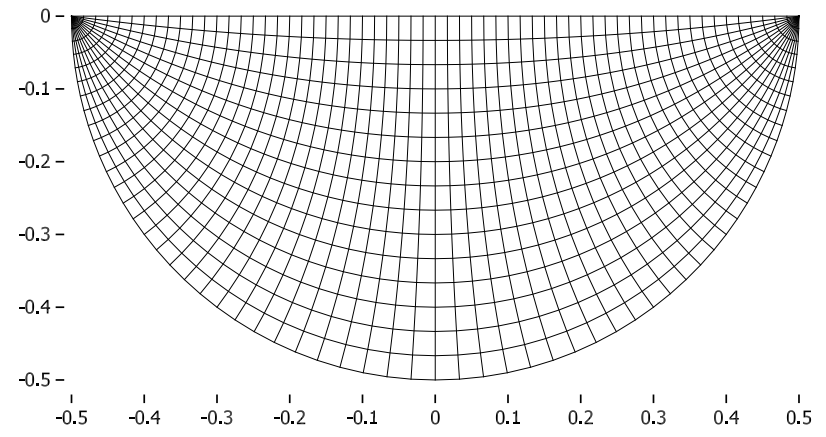

Figure 3. Body-fitted numerical mesh (1 out of every 10 grids is shown).

or by rearranging it, we have

$$
x^{2}+y^{2}+\frac{e^{2 c}+1}{e^{2 c}-1} x+\frac{1}{4}=0
$$

We note that the above equation defines a system of coaxial circles with their centres being on the $x$ axis as shown in Figure 2 The centre coordinates of these coaxial circles are given as $x=-\frac{1}{2} \frac{e^{2 c}+1}{e^{2 c}-1}$, $y=0$. Also, the radii of these circles are equal to $r=\frac{1}{2} \sqrt{\left(\frac{e^{2 c}+1}{e^{2 c}-1}\right)^{2}-1}$. We also note that when $c=0$, the radius of the circle becomes infinite, which is a perpendicular straight line at the origin.

Following the same procedure, for any value of the stream function, such that $\psi=c$, we have

$$
c=\arctan \frac{y}{x-\frac{1}{2}}-\arctan \frac{y}{x+\frac{1}{2}}
$$

By rearranging it, we get

$$
x^{2}+y^{2}-\frac{y}{\tan c}-\frac{1}{4}=0
$$

We note that the above equation defines a system of coaxial circles with their centres being on the $y$-axis as also shown in Figure 2. The centre coordinates of these coaxial circles are given as $x=0$, $y=\frac{1}{2} \tan c$. Also, the radii of these circles are equal to $r=\frac{1}{2} \sqrt{\frac{1}{(\tan c)^{2}}+1}$. We note that each of these circles passes through the location of the source and the sink, i.e., through the points $\left(-\frac{1}{2}, 0\right)$ and $\left(\frac{1}{2}, 0\right)$.

Using the equations given above, we can easily obtain the mesh points inside the semi-circular cavity. In order to keep the aspect ratio $\left(\frac{\Delta x}{\Delta y}\right)$ of the grid points in the mesh more or less around unity in majority of the domain, we used $600 \times 150$ grid points in the mesh. We divide the upper lid of the arc shaped cavity into 600 equal points and we calculate the values of the velocity potential function $(\phi=c)$ at these points. Similarly, we divide the vertical line at $x=0$ into 150 equal points and we calculate values of the stream function $(\psi=c)$ at these points. Then, the intersection points of these velocity potential function $(\phi)$ and stream function $(\psi)$ are obtained as the grid point locations. The body-fitted orthogonal mesh we used is given Figure 3

\subsection{Governing EQUations in the COMPUTATIONAL DOMAIN}

Since $\phi$ and $\psi$ provide a body-fitted orthogonal coordinate system for the considered semi-circular cavity geometry, the natural choice is to use $\phi$ and $\psi$ as the coordinate axes in the mapped domain. However, the value of $\phi$ at the corners of the arc shaped cavity (i.e., at the location of the source and the sink) is equal to $\pm \infty$. For this reason, it was not possible to use $\phi$ and $\psi$ as coordinate axes. Also, using $\phi$ and $\psi$ as coordinate axes (except at the the corner points) introduces extra difficulty in finite differencing of the spatial derivatives on a non-uniform mesh. In order to overcome this difficulty, we transform the curvilinear mesh in physical domain to a rectangular uniform mesh in computational domain with $\xi$ and $\eta$ coordinates where the grid spacing is uniform and equal to unity (i.e. $\Delta \xi=\Delta \eta=1$ ). The $x$ and $y$ derivatives are transformed to the computational domain as the following

$$
\begin{gathered}
f_{x}=\frac{y_{\eta} f_{\xi}-y_{\xi} f_{\eta}}{J} \\
f_{y}=\frac{-x_{\eta} f_{\xi}+x_{\xi} f_{\eta}}{J}
\end{gathered}
$$

where $f$ is any differentiable function and $J$ is the Jacobian of the transformation, which is defined as

$$
J=x_{\xi} y_{\eta}-x_{\eta} y_{\xi}
$$

In the computational $\xi-\eta$ domain, the governing streamfunction and vorticity equations are transformed as the following

$$
\frac{\alpha \psi_{\xi \xi}-2 \beta \psi_{\xi \eta}+\gamma \psi_{\eta \eta}+\sigma \psi_{\eta}+\tau \psi_{\xi}}{J^{2}}=\omega
$$

$$
\begin{aligned}
& \psi_{\eta} \omega_{\xi}-\psi_{\xi} \omega_{\eta}= \\
& \quad \frac{1}{R e} \frac{\alpha \omega_{\xi \xi}-2 \beta \omega_{\xi \eta}+\gamma \omega_{\eta \eta}+\sigma \omega_{\eta}+\tau \omega_{\xi}}{J}
\end{aligned}
$$

where the coefficients in these equations $(\alpha, \beta, \gamma, \sigma$, $\tau)$ are defined as

$$
\begin{gathered}
\alpha=x_{\eta}^{2}+y_{\eta}^{2} \\
\beta=x_{\xi} x_{\eta}+y_{\xi} y_{\eta} \\
\gamma=x_{\xi}^{2}+y_{\xi}^{2} \\
\sigma=\frac{y_{\xi}(D x)-x_{\xi}(D y)}{J} \\
\tau=\frac{x_{\eta}(D y)-y_{\eta}(D x)}{J}
\end{gathered}
$$

where $D x$ and $D y$ are

$$
\begin{gathered}
D x=\alpha x_{\xi \xi}-2 \beta x_{\xi \eta}+\gamma x_{\eta \eta} \\
D y=\alpha y_{\xi \xi}-2 \beta y_{\xi \eta}+\gamma y_{\eta \eta}
\end{gathered}
$$

Since we are only interested in the stationary solutions rather than the transient solutions in this study, 


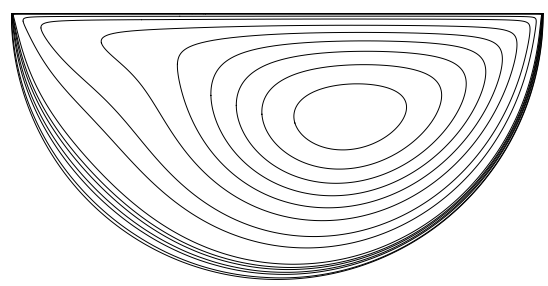

(A) $\cdot R e=500$

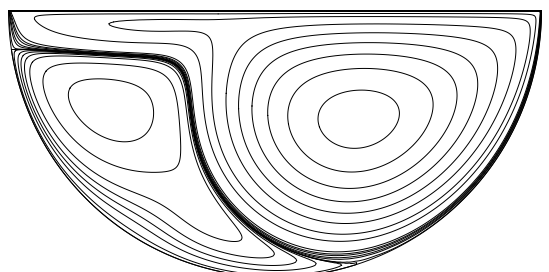

(D) $\cdot R e=3000$

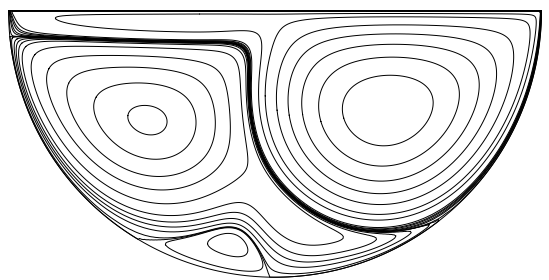

(G) $\cdot R e=6600$

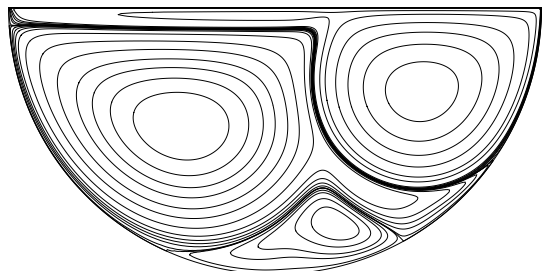

(J) $\cdot R e=15000$

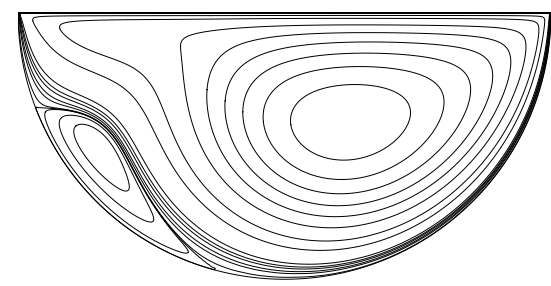

(в) $\cdot R e=1000$

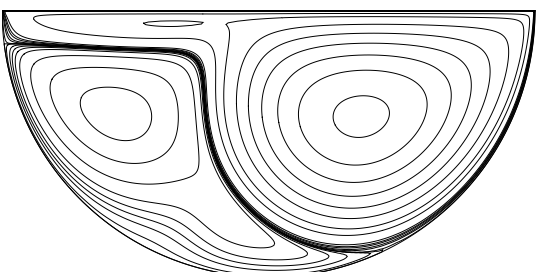

(E) $\cdot R e=4000$

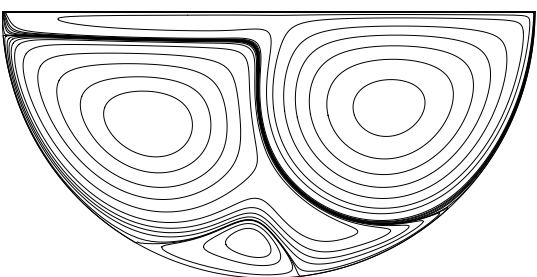

(н) $. R e=8000$

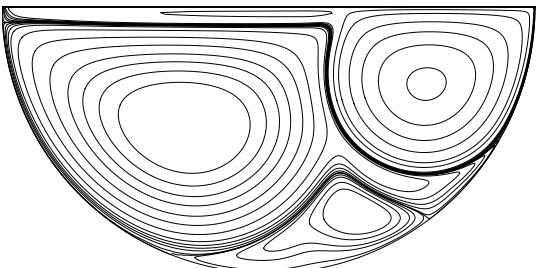

(к) . $R e=20000$

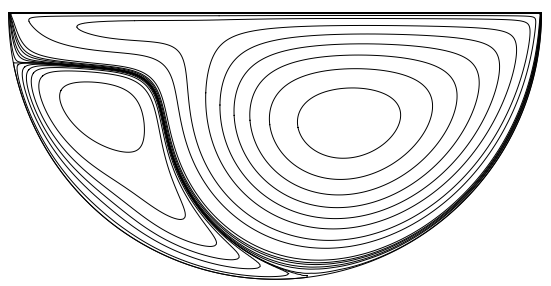

(C) $\cdot R e=2000$

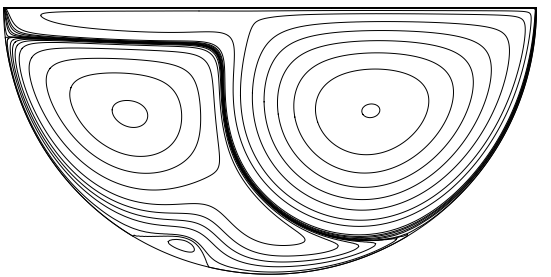

(F) $\cdot R e=5000$

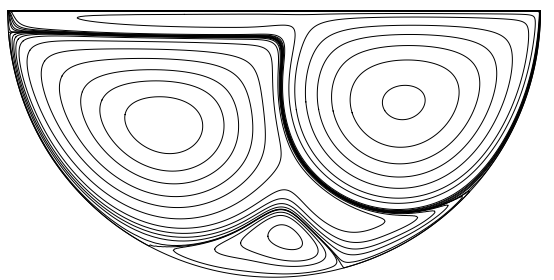

(I) $\cdot R e=10000$

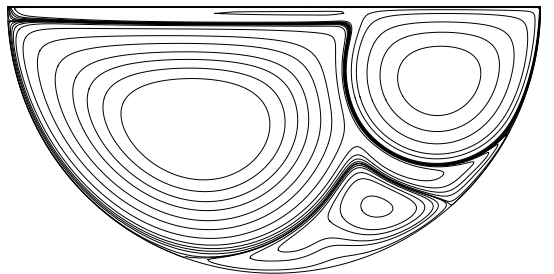

(L) $\cdot R e=24000$

FiguRE 4. Streamline contours of semi-circular cavity flow as Reynolds number increases.

we used the Successive Over Relaxation (SOR) method in order to solve the governing steady streamfunction and vorticity equations numerically. These governing equations are numerically solved in the computational domain using 3 point second order accurate central differencing, $\mathcal{O}\left(\Delta \xi^{2}, \Delta \eta^{2}\right)$.

\subsection{MAPPING TRANSFORMATION METRICS}

We calculate the mapping transformation metrics $\left(x_{\xi}\right.$, $\left.x_{\eta}, y_{\xi}, y_{\eta}\right)$ numerically using finite difference. The mapping transformation metrics appear explicitly in the coefficients $(\alpha, \beta, \gamma, \sigma, \tau)$ of the governing streamfunction and vorticity equations 13 and 14 . In order to minimize the effect of the finite difference approximation errors associated with numerically calculated mapping transformation metrics on the numerical solution of the governing flow equations, we decided to use fourth order accurate differencing, $\mathcal{O}\left(\Delta \xi^{4}, \Delta \eta^{4}\right)$, in calculating the mapping metrics, which is higher than the accuracy of the streamfunction and vorticity finite difference equations. With high order accurate finite difference approximation for the mapping trans- formation metrics, we obtain more accurate numerical solutions for streamfunction and vorticity equations. We note that since we calculate the mapping metrics only once, the increase in the computational effort when using higher accuracy for the mapping metrics is insignificant as compared to the computational effort used for solving the governing streamfunction and vorticity equations. At the interior points of the computational domain, the mapping metrics are calculated using the following 5 point central fourth order finite difference equation

$$
\left(f_{\eta}\right)_{i, j}=\frac{f_{i, j-2}-8 f_{i, j-1}+8 f_{i, j+1}-f_{i, j+2}}{12}
$$

where again, $f$ is any differentiable function, which, in our case, can be $x$ or $y$ and the subscripts $i$ and $j$ denote the grid index in $\xi$ and $\eta$ directions, respectively. Using the same finite difference equation, the transformation metrics for $\xi$-direction $\left(f_{\xi}\right)$ are calculated similarly. Near the boundaries of the computational domain, at the first grid points above the wall, the mapping metrics are calculated using the following 5 


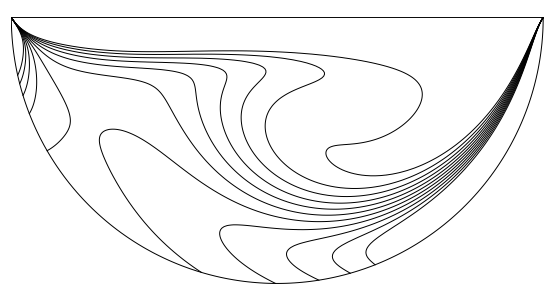

(A) . $R e=500$

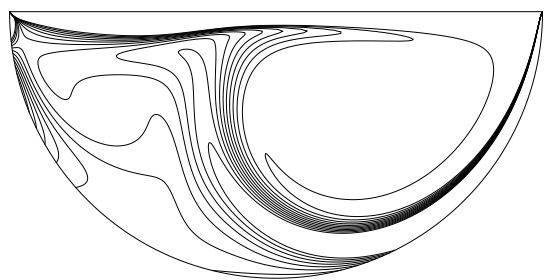

(D) . $R e=3000$

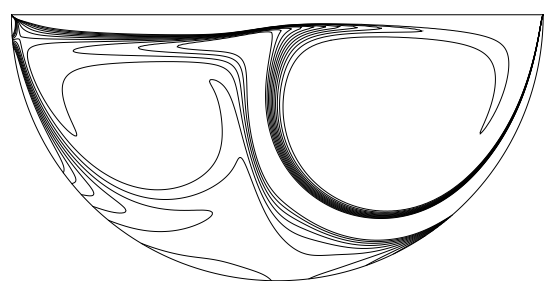

(G) $\cdot R e=6600$

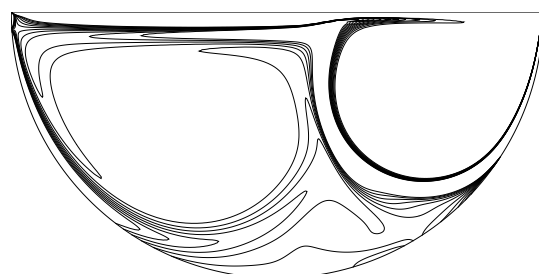

(J) $\cdot R e=15000$

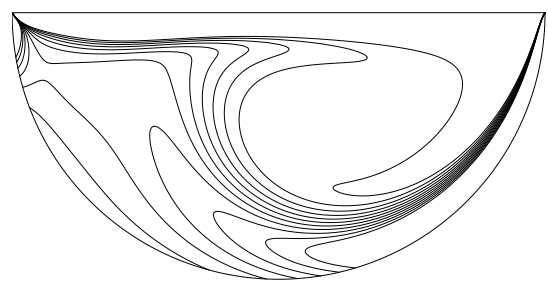

(в) $\cdot R e=1000$

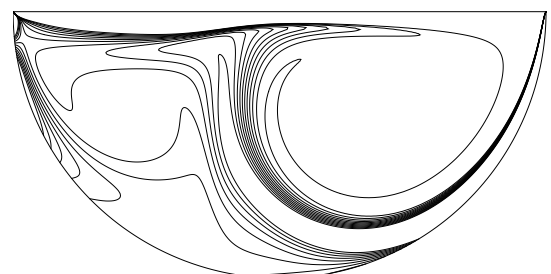

(E) . $R e=4000$

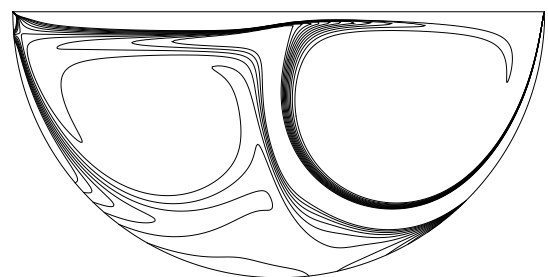

(н) . $R e=8000$

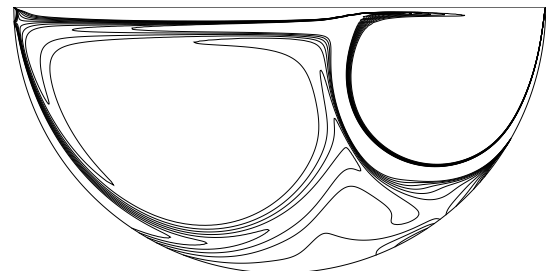

(к) $\cdot R e=20000$

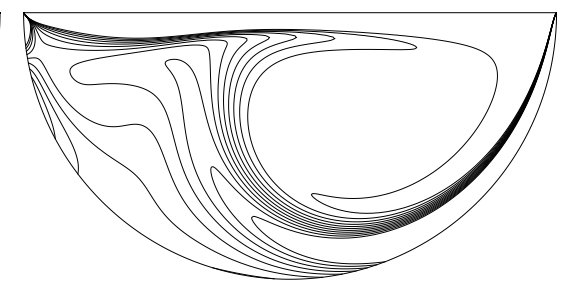

(C) $\cdot R e=2000$

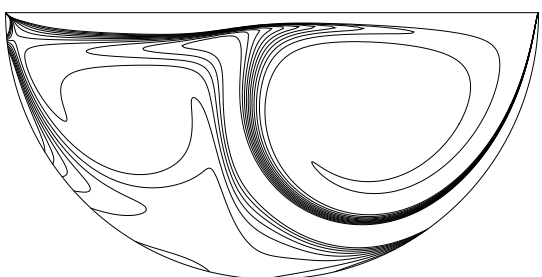

(F) . $R e=5000$

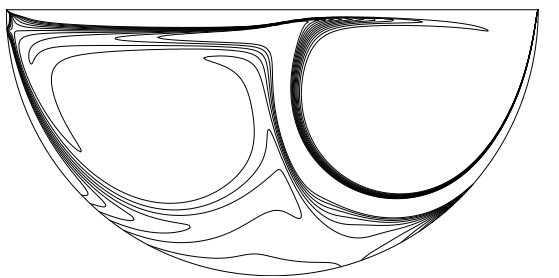

(I) $. R e=10000$

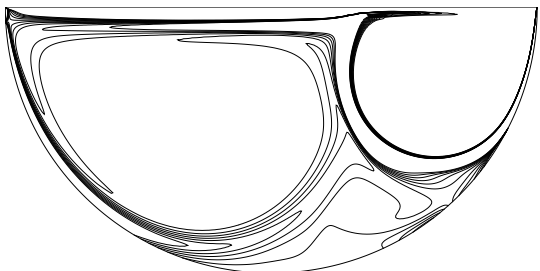

(L) $\cdot R e=24000$

FIGURE 5. Vorticity contours of semi-circular cavity flow as Reynolds number increases.

point one-sided fourth order finite difference equation

$$
\left(f_{\eta}\right)_{i, 1}=\frac{-3 f_{i, 0}-10 f_{i, 1}+18 f_{i, 2}-6 f_{i, 3}+f_{i, 4}}{12}
$$

where the subscripts 0 refer to the wall grid points and 1,2,3 and 4 refer to grid points above the wall in the order. Similarly, on the boundaries of the computational domain at the points on the wall, the mapping metrics are calculated using the following 5 point one-sided fourth order finite difference equation

$$
\left(f_{\eta}\right)_{i, 0}=\frac{-25 f_{i, 0}+48 f_{i, 1}-36 f_{i, 2}+16 f_{i, 3}-3 f_{i, 4}}{12}
$$

Using the above finite difference equations, the transformation metrics $x_{\xi}, x_{\eta}, y_{\xi}, y_{\eta}$ are calculated with fourth order accuracy $\mathcal{O}\left(\Delta \xi^{4}, \Delta \eta^{4}\right)$.

\subsection{WALL BOUNDARY CONDITIONS}

Using the velocity of the top moving lid and simplifying terms, at the top wall, we obtain

$$
U=\psi_{y}=\frac{-x_{\eta} \psi_{\xi}+x_{\xi} \psi_{\eta}}{J}=\frac{x_{\xi} \psi_{\eta}}{J}=1
$$

Simplifying the streamfunction equation and also substituting the above equation, at the grid points on the top moving wall, the vorticity value is obtained as the following

$$
\omega(i, 0)=\frac{\gamma}{J^{2}}\left(2 \psi(i, 1)+2 \frac{J}{x_{\xi}}\right)
$$

Similarly, on the bottom curved wall, the vorticity value is obtained as the following

$$
\omega(i, 0)=2 \frac{\gamma}{J^{2}} \psi(i, 1)
$$

\section{Results AND Discussion}

As convergence criteria in our simulations, we use the change in the streamfunction and vorticity variables between two consecutive iterations, which is normalized by the previous values defined as the following

$$
\operatorname{Residual}_{\psi}=\max \left(\left|\frac{\psi_{i, j}^{k+1}-\psi_{i, j}^{k}}{\psi_{i, j}^{k}}\right|\right)
$$




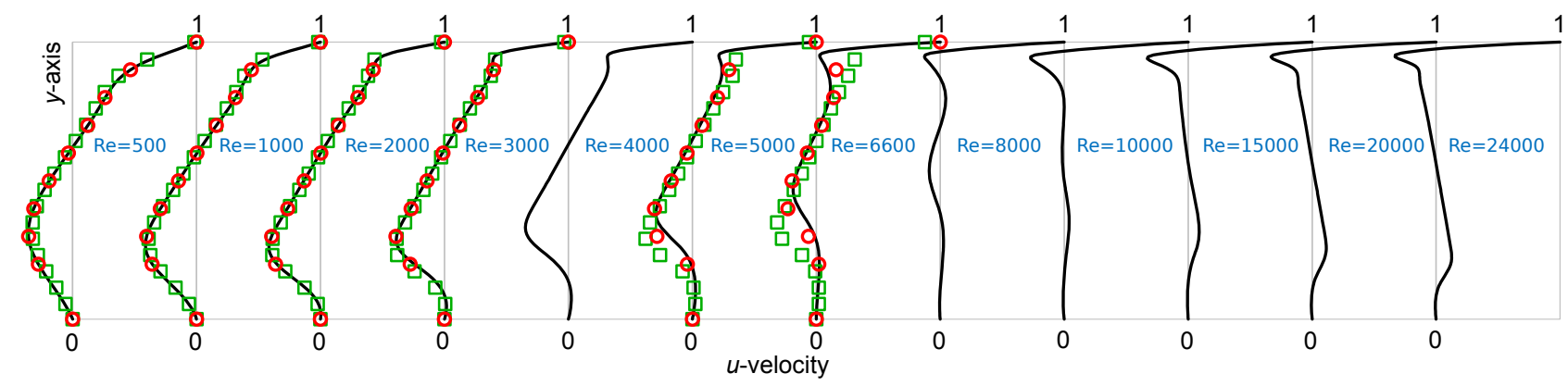

FiguRE 6 . The $u$-velocity profiles along the vertical line at $x=0$ at various Reynolds numbers, o symbols [10], $\square$ symbols 13 .

$$
\operatorname{Residual}_{\omega}=\max \left(\left|\frac{\omega_{i, j}^{k+1}-\omega_{i, j}^{k}}{\omega_{i, j}^{k}}\right|\right)
$$

where max denotes the maximum value in the solution domain and the superscript denotes the iteration number. In our numerical solutions at the convergence both Residual $_{\psi}$ and Residual $\omega$ are less than $10^{-6}$. This means that at the convergence, the streamfunction and vorticity values change less than one millionth of their values between two consecutive iterations at a grid point in the computational domain as the maximum in absolute value and even less in the rest of the grid points in the computational domain.

For the mesh generation and also for the numerical solution of the governing equations, computer codes in $\mathrm{C}++$ programming language are written and using these codes, we numerically solve the incom-

\begin{tabular}{llll}
\hline \multirow{2}{*}{$R e$} & & $\psi_{\min }$ & $(x, y)$ \\
\hline \multirow{2}{*}{1000} & present study & -0.07808 & $(0.6193,-0.2030)$ \\
& Glowinski et al. [10] & $-0,0779$ & $(0.6214,-0.2030)$ \\
& Yang et al. [12] & -0.0775 & $(0.6201,-0.2060)$ \\
& Yu et al. [14] & -0.0779 & $(0.6225,-0.2044)$ \\
2000 & present study & -0.07674 & $(0.6359,-0.2062)$ \\
& Glowinski et al. [10] & -0.0763 & $(0.6359,-0.2052)$ \\
& Yang et al. [12] & -0.076 & $(0.6376,-0.2061)$ \\
& Yu et al. [14] & -0.0764 & $(0.6367,-0.2046)$ \\
3000 & present study & -0.07461 & $(0.6551,-0.2020)$ \\
& Glowinski et al. [10] & -0.0742 & $(0.6514,-0.2027)$ \\
& Yang et al. [12] & -0.0737 & $(0.6553,-0.2041)$ \\
& Yu et al. [14] & -0.0740 & $(0.6636,-0.1993)$ \\
5000 & present study & -0.07026 & $(0.6891,-0.1931)$ \\
& Glowinski et al. [10] & -0.07 & $(0.6833,-0.1936)$ \\
& Yang et al. [12] & -0.069 & $(0.6846,-0.1963)$ \\
& Yu et al. [14] & -0.0691 & $(0.7055,-0.1863)$ \\
& Mercan et al. [15] & -0.0694 & $(0.7132,-0.1825)$ \\
6600 & present study & -0.06718 & $(0.7096,-0.1868)$ \\
& Glowinski et al. [10] & -0.067 & $(0.7009,-0.1891)$ \\
Yang et al. [12] & -0.0657 & $(0.7002,-0.1905)$ \\
& Yu et al. [14] & -0.0652 & $(0.7353,-0.1745)$ \\
& Mercan et al. [15] & -0.0656 & $(0.7464,-0.1704)$ \\
\hline
\end{tabular}

TABle 1. Comparison of the minimum streamfunction and location of center of the primary vortex. pressible viscous flow in a semi-circular cavity. We start solving the considered flow for Reynolds number of 500. Then, we progressively increase the Reynolds number by using the previous Reynolds number solution as the initial guess for the next highest Reynolds number. With this approach, we obtain numerical solutions of the wall-driven semi-circular cavity flow up to Reynolds number of 24000 .

The streamfunction and vorticity contours presented in Figure 4 and Figure 5 show the change in the flow topology in a semi-circular cavity as the Reynolds number increases. From Figure 4 and Figure 5, we can see that between $R e=500$ and $R e=1000$ and $R e=4000$ and $R e=5000$, a secondary and a tertiary vortex, respectively, appear in the flow field. We can also see that as the Reynolds number increases, the size of both the secondary and the tertiary vortex increases where as the size of the primary vortex decreases.

Glowinski et al. 10], Yang et al. [12, Yu et al. [14] and Mercan et al. [15] have presented detailed data from the flow field. In Table 1 we compare the present results of the minimum streamfunction value and its location in the semi-circular cavity geometry with the results presented in [10, 12, 14, 15]. In their study, Glowinski et al. 10] also presented the detachment angles of the secondary and tertiary vortex measured counterclockwise from the top lid as shown in Figure 1 . On the wall boundary, the skin-friction coefficient is

\begin{tabular}{ccccc}
\hline$R e$ & $\theta_{S V}^{\text {begin }}$ & $\theta_{S V}^{\text {end }}$ & $\theta_{T V}^{\text {begin }}$ & $\theta_{T V}^{\text {end }}$ \\
\hline \multicolumn{5}{c}{ Present study } \\
1000 & 20.83 & 74.83 & - & - \\
2000 & 10.87 & 97.01 & - & - \\
3000 & 8.38 & 107.94 & - & - \\
5000 & 6.39 & 121.23 & 59.12 & 76.44 \\
\hline \multicolumn{5}{c}{ Glowinski et al. [10] } \\
1000 & 21.42 & 71.49 & - & - \\
2000 & 10.78 & 94.34 & - & - \\
3000 & 7.77 & 104.53 & - & - \\
5000 & 6.70 & 117.09 & 57.54 & 73.84 \\
\hline
\end{tabular}

TABLE 2. Comparison of the detachment angles of the secondary and tertiary vortex. 


\begin{tabular}{|c|c|c|c|c|c|c|c|c|c|c|c|c|}
\hline \multirow[b]{2}{*}{$\mathrm{y}$} & \multicolumn{12}{|c|}{$R e$} \\
\hline & 500 & 1000 & 2000 & 3000 & 4000 & 5000 & 6600 & 8000 & 10000 & 15000 & 20000 & 24000 \\
\hline 0 & 1 & 1 & 1 & 1 & 1 & 1 & 1 & 1 & 1 & 1 & 1 & 1 \\
\hline-0.0333 & 0.6094 & 0.5398 & 0.4523 & 0.3901 & 0.3166 & 0.2239 & 0.0453 & -0.1074 & -0.2528 & -0.3103 & -0.2870 & -0.2660 \\
\hline-0.0667 & 0.3734 & 0.3962 & 0.3932 & 0.3531 & 0.2973 & 0.2314 & 0.1075 & 0.0043 & -0.0606 & -0.0828 & -0.1135 & -0.1315 \\
\hline-0.1 & 0.2636 & 0.3161 & 0.3005 & 0.2609 & 0.2175 & 0.1745 & 0.1056 & 0.0437 & -0.0042 & -0.0630 & -0.0937 & -0.1030 \\
\hline-0.1333 & 0.1729 & 0.2151 & 0.1954 & 0.1631 & 0.1299 & 0.0998 & 0.0590 & 0.0247 & -0.0020 & -0.0478 & -0.0648 & -0.0716 \\
\hline-0.1667 & 0.0733 & 0.1081 & 0.0955 & 0.0712 & 0.0453 & 0.0219 & -0.0082 & -0.0221 & -0.0118 & -0.0308 & -0.0390 & -0.0433 \\
\hline-0.2 & -0.0321 & 0.0040 & 0.0012 & -0.0169 & -0.0379 & -0.0581 & -0.0830 & -0.0693 & -0.0166 & -0.0120 & -0.0139 & -0.0161 \\
\hline-0.2333 & -0.1371 & -0.0962 & -0.0894 & -0.1028 & -0.1212 & -0.1407 & -0.1534 & -0.0890 & -0.0092 & 0.0109 & 0.0120 & 0.0108 \\
\hline-0.2667 & -0.2346 & -0.1940 & -0.1778 & -0.1879 & -0.2060 & -0.2250 & -0.1873 & -0.0627 & 0.0108 & 0.0383 & 0.0386 & 0.0374 \\
\hline-0.3 & -0.3130 & -0.2902 & -0.2665 & -0.2746 & -0.2922 & -0.2897 & -0.1400 & -0.0128 & 0.0332 & 0.0676 & 0.0650 & 0.0634 \\
\hline-0.3333 & -0.3546 & -0.3745 & -0.3560 & -0.3592 & -0.3471 & -0.2547 & -0.0488 & 0.0197 & 0.0393 & 0.0886 & 0.0915 & 0.0884 \\
\hline-0.3667 & -0.3433 & -0.4120 & -0.4148 & -0.3813 & -0.2693 & -0.1121 & 0.0095 & 0.0256 & 0.0256 & 0.0744 & 0.1134 & 0.1144 \\
\hline-0.4 & -0.2785 & -0.3593 & -0.3556 & -0.2464 & -0.0963 & -0.0101 & 0.0250 & 0.0167 & 0.0084 & 0.0287 & 0.0827 & 0.1189 \\
\hline-0.4333 & -0.1811 & -0.2253 & -0.1768 & -0.0668 & 0.0027 & 0.0228 & 0.0182 & 0.0039 & -0.0038 & 0.0000 & 0.0160 & 0.0384 \\
\hline-0.4667 & -0.0809 & -0.0836 & -0.0309 & 0.0122 & 0.0238 & 0.0225 & 0.0072 & -0.0055 & -0.0087 & -0.0058 & -0.0060 & -0.0046 \\
\hline-0.5 & 0 & 0 & 0 & 0 & 0 & 0 & 0 & 0 & 0 & 0 & 0 & 0 \\
\hline
\end{tabular}

TABLE 3 . Selected $u$-velocity values along $x=0$ line.

defined as

$$
C_{f}=\frac{\tau_{w}}{\rho U^{2}}
$$

where $\tau_{w}=\left.\mu \frac{\partial u}{\partial y}\right|_{w}$. At the detachment points on the wall, the skin-friction coefficient will be zero. In our study, by calculating the skin-friction coefficient, we have located the detachment points in the lower circular wall of the semi-circular cavity. In Table 2 , we compare our results of the detachment angles of the secondary and tertiary vortex with the results of Glowinski et al. [10. Present results in Table 1 and Table 2 are in good agreement with the results of Glowinski et al. [10, Yang et al. [12, Yu et al. [14] and Mercan et al. [15].

In Figure6, we plot the computed $u$-velocity profiles along the vertical line at the mid of the semi-circular cavity, i.e., at $x=0$ line, at various Reynolds numbers. In the same figure, the velocity profiles of Glowinski et al. 10] and Ding et al. [13] are also given with red and green symbols, respectively. As seen in Figure6, at low Reynolds numbers, our computed $u$-velocity profiles are in good agreement with the $u$-velocity profiles of Glowinski et al. [10] and Ding et al. [13, where as at large Reynolds numbers, our velocity profiles deviate from those of Glowinski et al. [10] and Ding et al. 13. We believe that due to the fine mesh used in the present study, our results are more accurate. In Table 3, we tabulate selected values of the $u$-velocity values along $x=0$ line at various Reynolds numbers for future references.

In Table 4 and Table 5 , we present the streamfunction and vorticity values at the centres of the vortices together with the centre locations and also the detachment angles of the secondary and tertiary vortex, respectively, for all the Reynolds numbers considered in this study for benchmark purposes.

In order to see the behaviour of the detachment points better, in Figure 7, we plot the detachment angles given in Table 4 as a function of the Reynolds number. From Figure 7, we can see that while $\theta_{S V}^{\text {begin }}$ seems to converge to a value at large Reynolds num-

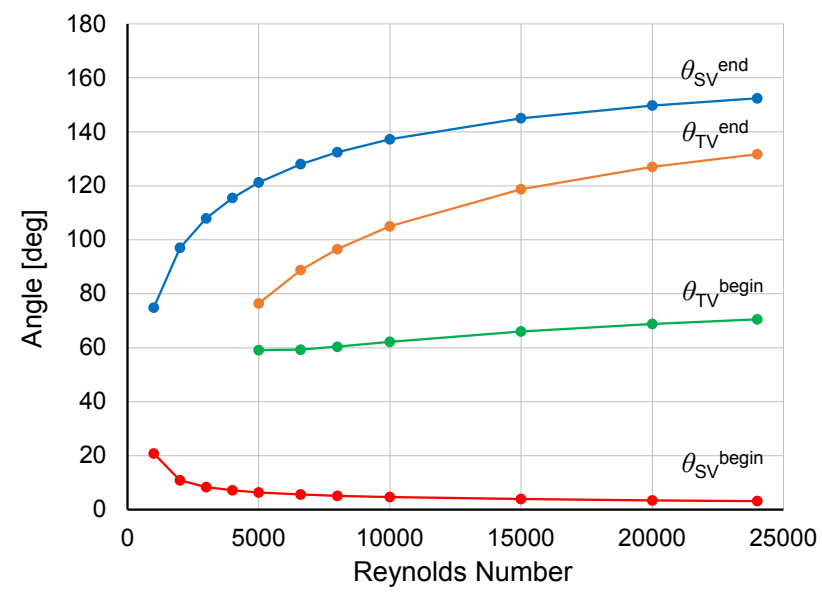

Figure 7. Detachment angles of the secondary and tertiary vortex as a function of the Reynolds number.

bers, $\theta_{S V}^{e n d}, \theta_{T V}^{\text {begin }}$ and $\theta_{T V}^{\text {end }}$ are still increasing as the Reynolds number increases. This states that the solution of the semi-circular cavity flow is still changing even at the highest Reynolds number considered in the present study (i.e. $R e=24000$ ).

At large Reynolds number, the Batchelor's model (29) states that the steady laminar flow with closed streamlines moves like a solid body with a uniform vorticity at the inviscid vortex. Erturk [2] showed that in the driven square cavity flow at large Reynolds numbers in the primary vortex, the vorticity is almost uniform such that the core fluid rotates like a solid body in agreement with the Batchelor's model ([29]), also, at the primary vortex centre, the vorticity value converges to the limiting value analytically calculated by Burggraf [30]. In the case of the driven semicircular cavity flow at large Reynolds numbers, from Figure 4 and Figure 7 , we can see that the sizes of the primary, secondary and tertiary vortices are still changing significantly even at the highest considered Reynolds number of $R e=24000$, while the size of the primary vortex decreases, the size of the secondary and tertiary vortices increases. This indicates that 


\begin{tabular}{cccccc}
\hline$R e$ & & $\psi$ & $\omega$ & $x$ & $y$ \\
\hline 500 & PV & $-7.6674 \mathrm{E}-02$ & 4.73550 & 0.1334 & -0.1940 \\
1000 & PV & $-7.8084 \mathrm{E}-02$ & 4.53861 & 0.1193 & -0.2030 \\
& SV & $4.1296 \mathrm{E}-04$ & -0.58656 & -0.3456 & -0.2570 \\
2000 & PV & $-7.6735 \mathrm{E}-02$ & 4.57210 & 0.1359 & -0.2062 \\
& SV & $3.8975 \mathrm{E}-03$ & -1.37969 & -0.3250 & -0.1817 \\
3000 & PV & $-7.4606 \mathrm{E}-02$ & 4.70449 & 0.1551 & -0.2020 \\
& SV & $6.5343 \mathrm{E}-03$ & -1.48358 & -0.3096 & -0.1836 \\
4000 & PV & $-7.2385 \mathrm{E}-02$ & 4.86208 & 0.1722 & -0.1978 \\
& SV & $8.6265 \mathrm{E}-03$ & -1.47665 & -0.2829 & -0.1933 \\
5000 & PV & $-7.0263 \mathrm{E}-02$ & 5.02531 & 0.1891 & -0.1931 \\
& SV & $1.0289 \mathrm{E}-02$ & -1.47578 & -0.2609 & -0.1986 \\
& TV & $-1.5422 \mathrm{E}-05$ & 0.13901 & -0.1629 & -0.4461 \\
6600 & PV & $-6.7180 \mathrm{E}-02$ & 5.28290 & 0.2096 & -0.1868 \\
& SV & $1.2349 \mathrm{E}-02$ & -1.46225 & -0.2396 & -0.2052 \\
& TV & $-1.5500 \mathrm{E}-04$ & 0.37152 & -0.0828 & -0.4365 \\
8000 & PV & $-6.4793 \mathrm{E}-02$ & 5.50007 & 0.2252 & -0.1786 \\
& SV & $1.3747 \mathrm{E}-02$ & -1.44986 & -0.2236 & -0.2086 \\
& TV & $-3.0604 \mathrm{E}-04$ & 0.47522 & -0.0321 & -0.4323 \\
10000 & PV & $-6.1823 \mathrm{E}-02$ & 5.79482 & 0.2432 & -0.1720 \\
& SV & $1.5309 \mathrm{E}-02$ & -1.43214 & -0.2037 & -0.2128 \\
& TV & $-4.8690 \mathrm{E}-04$ & 0.56585 & 0.0230 & -0.4261 \\
15000 & PV & $-5.6092 \mathrm{E}-02$ & 6.45364 & 0.2757 & -0.1561 \\
& SV & $1.7854 \mathrm{E}-02$ & -1.39370 & -0.1747 & -0.2220 \\
& TV & $-7.8118 \mathrm{E}-04$ & 0.67408 & 0.1123 & -0.4074 \\
20000 & PV & $-5.1952 \mathrm{E}-02$ & 7.02646 & 0.2953 & -0.1447 \\
& SV & $1.9332 \mathrm{E}-02$ & -1.36248 & -0.1543 & -0.2243 \\
& TV & $-9.7761 \mathrm{E}-04$ & 0.73133 & 0.1649 & -0.3892 \\
24000 & PV & $-4.9336 \mathrm{E}-02$ & 7.44717 & 0.3088 & -0.1382 \\
& SV & $2.0103 \mathrm{E}-02$ & -1.34078 & -0.1425 & -0.2272 \\
& TV & $-1.0986 \mathrm{E}-03$ & 0.75759 & 0.1919 & -0.3758 \\
\hline & & & & & \\
& & & & & \\
5
\end{tabular}

TABLE 4. Streamfunction, vorticity at the centres of vortices and $(x, y)$ locations at different Reynolds numbers.

even at $R e=24000$, the flow is still evolving and the flow quantities are not converging to a value asymptotically. However, in the case of the square driven cavity in Erturk [2], the change in the the primary vortex's size or the change in the flow quantities at the centre of the primary vortex becomes very small as the Reynolds number increases to high values converging to a stable value. In order to see the behaviour of the driven semi-circular cavity flow at large Reynolds numbers better, in Figure 8 we plot the vorticity value at the centre of the primary vortex with respect to the Reynolds numbers, which are tabulated in Table 3 . From Figure 8, we can see that the vorticity value at the primary vortex centre continuously increases almost linearly with respect to the Reynolds number without converging to a value even at $R e=24000$. Present results show that at large Reynolds numbers, the driven semi-circular cavity flow does not agree with the Batchelor's model ([29]).

\section{Conclusion}

In this study we have presented stationary solutions of an incompressible viscous flow in a wall-driven semicircular cavity at large Reynolds numbers. The governing equations are numerically solved up to $R e=24000$

\begin{tabular}{crrcc}
\hline$R e$ & $\theta_{S V}^{\text {begin }}$ & $\theta_{S V}^{\text {end }}$ & $\theta_{T V}^{\text {begin }}$ & $\theta_{T V}^{\text {end }}$ \\
\hline 1000 & 20.83 & 74.83 & - & - \\
2000 & 10.87 & 97.01 & - & - \\
3000 & 8.38 & 107.94 & - & - \\
4000 & 7.16 & 115.52 & - & - \\
5000 & 6.39 & 121.23 & 59.12 & 76.44 \\
6600 & 5.61 & 128.01 & 59.28 & 88.75 \\
8000 & 5.14 & 132.45 & 60.38 & 96.53 \\
10000 & 4.66 & 137.28 & 62.15 & 105.01 \\
15000 & 3.90 & 145.04 & 65.99 & 118.75 \\
20000 & 3.43 & 149.75 & 68.79 & 127.04 \\
24000 & 3.16 & 152.44 & 70.52 & 131.72 \\
\hline
\end{tabular}

TABLE 5. Flow detachment angles of the secondary $(\mathrm{SV})$ and tertiary (TV) vortices at different Reynolds numbers.

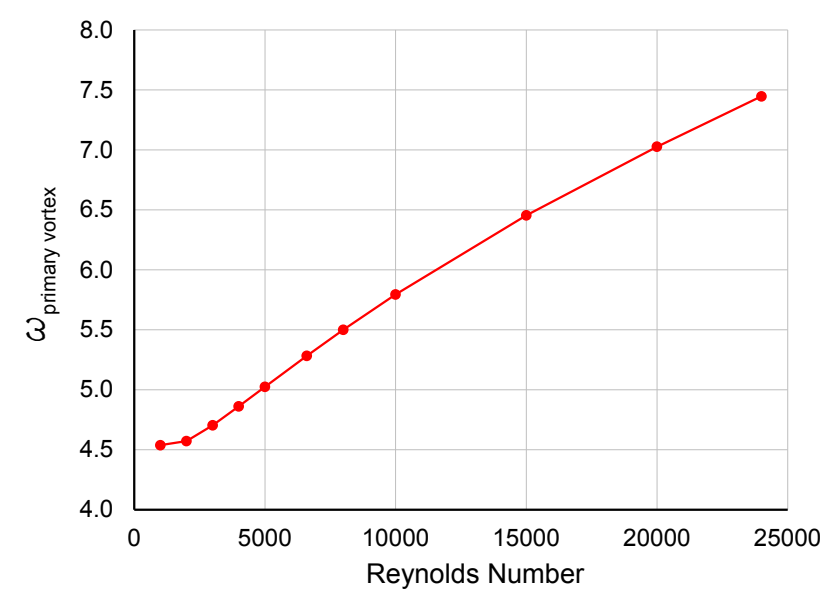

FiguRE 8. Variation of the vorticity value as a function of the Reynolds number at the centre of the primary vortex.

with using a body-fitted mesh obtained by a conformal mapping. Our numerical solutions are in good agreement with the numerical solutions found in the literature. Our computations indicate that as the Reynolds number increases up to $R e=24000$, the size of the secondary and tertiary vortex increases continuously whereas the size of the primary vortex decreases continuously. Present numerical solutions show that the vorticity value at the centre of the primary vortex increases almost linearly at large Reynolds numbers, up to the highest considered Reynolds number of 24000 in this study, stating that Batchelor's mean-square law is not valid for the wall-driven semi-circular cavity flow.

\section{REFERENCES}

[1] U. Ghia, K. Ghia, C. Shin. High-re solutions for incompressible flow using the navier-stokes equations and a multigrid method. Journal of Computational Physics 48(3):387-411, 1982. https://doi.org/10.1016/0021-9991(82)90058-4.

[2] E. Erturk. Discussions on driven cavity flow. International Journal for Numerical Methods in Fluids 
60(3):275-294, 2009 https://doi.org/10.1002/fld.1887

[3] B. Bastl, M. Brandner, J. Egermaier, et al. Numerical simulation of lid-driven cavity flow by isogeometric analysis. Acta Polytechnica 61(SI):33-48, 2021. https://doi.org/10.14311/AP.2021.61.0033.

[4] I. Demirdžić, Ž. Lilek, M. Perić. Fluid flow and heat transfer test problems for non-orthogonal grids: Bench-mark solutions. International Journal for Numerical Methods in Fluids 15(3):329-354, 1992. https://doi.org/10.1002/fld.1650150306

[5] E. Erturk, B. Dursun. Numerical solutions of 2-D steady incompressible flow in a driven skewed cavity. ZAMM - Journal of Applied Mathematics and Mechanics 87(5):377-392, 2007. https://doi.org/10.1002/zamm.200610322

[6] C. Ribbens, L. Watson, C.-Y. Wang. Steady viscous flow in a triangular cavity. Journal of Computational Physics 112(1):173-181, 1994. https://doi.org/10.1006/jcph.1994.1090

[7] P. Gaskell, H. Thompson, M. Savage. A finite element analysis of steady viscous flow in triangular cavities. Proceedings of the Institution of Mechanical Engineers, Part C: Journal of Mechanical Engineering Science 213(3):263-276, 1999. https://doi.org/10.1243/0954406991522635.

[8] E. Erturk, O. Gokcol. Fine grid numerical solutions of triangular cavity flow. The European Physical Journal Applied Physics 38(1):97-105, 2007. https://doi.org/10.1051/epjap:2007057

[9] W. McQuain, C. Ribbens, C.-Y. Wang, L. Watson. Steady viscous flow in a trapezoidal cavity. Computers \& Fluids 23(4):613-626, 1994. https://doi.org/10.1016/0045-7930(94)90055-8

[10] R. Glowinski, G. Guidoboni, T.-W. Pan. Wall-driven incompressible viscous flow in a two-dimensional semi-circular cavity. Journal of Computational Physics 216(1):76-91, 2006. https://doi.org/10.1016/j.jcp.2005.11.021

[11] F. Yang, X. Shi, X. Guo, Q. Sai. MRT lattice Boltzmann schemes for high Reynolds number flow in two-dimensional lid-driven semi-circular cavity. Energy Procedia 16:639-644, 2012. https://doi.org/10.1016/j.egypro.2012.01.103.

[12] F. Yang, L. Liu, X. Shi, X. Guo. Lattice BGK study on flow pattern in two-dimensional wall-driven semi-circular cavity. Advanced Materials Research 354-355:549-598, 2011. https://doi.org/10.4028/ WWW.scientific.net/AMR.354-355.594.

[13] L. Ding, W. Shi, H. Luo, H. Zheng. Investigation of incompressible flow within $1 / 2$ circular cavity using lattice Boltzmann method. International Journal for Numerical Methods in Fluids 60(8):919-936, 2009. https://doi.org/10.1002/fld.1925.

[14] P. Yu, Z. Tian. A compact scheme for the streamfunction-velocity formulation of the $2 \mathrm{D}$ steady incompressible Navier-Stokes equations in polar coordinates. Journal of Scientific Computing 56:165-189, 2013. https://doi.org/10.1007/s10915-012-9667-7
[15] H. Mercan, K. Atalık. Vortex formation in lid-driven arc-shape cavity flows at high Reynolds numbers. European Journal of Mechanics - B/Fluids 28(1):61-71, 2009. https: //doi.org/10.1016/j.euromechflu.2008.02.001.

[16] C. Migeon, A. Texier, G. Pineau. Effects of lid-driven cavity shape on the flow establishment phase. Journal of Fluids and Structures 14(4):469-488, 2000. https://doi.org/10.1006/jfls.1999.0282.

[17] E. Erturk, O. Gokcol. Numerical solutions of steady incompressible flow around a circular cylinder up to Reynolds number 500. International Journal of Mechanical Engineering and Technology $\mathbf{9}(10): 1368-1378,2018$.

[18] C. Christov, R. Marinova, T. Marinov. Identifying the stationary viscous flows around a circular cylinder at high Reynolds numbers. Lecture Notes in Computer Science 4818:175-183, 2008. https://doi .org/10.1007/978-3-540-78827-0_18.

[19] H. Schlichting, K. Gersten. Boundary Layer Theory (8th revised and enlarged edn). Springer-Verlag Berlin Heidelberg, New York, USA, 2000.

[20] F. Smith. A structure for laminar flow past a bluff body at high Reynolds number. Journal of Fluid Mechanics 155:175-191, 1985. https://doi.org/10.1017/S0022112085001768.

[21] D. Peregrine. A note on the steady high-Reynolds-number flow about a circular cylinder. Journal of Fluid Mechanics 157:493-500, 1985. https://doi.org/10.1017/S0022112085002464.

[22] B. Fornberg. A numerical study of steady viscous flow past a circular cylinder. Journal of Fluid Mechanics 98(4):819-855, 1980. https://doi.org/10.1017/S0022112080000419.

[23] B. Fornberg. Steady viscous flow past a circular cylinder up to Reynolds number 600. Journal of Computational Physics 61(2):297-320, 1985. https://doi.org/10.1016/0021-9991(85)90089-0.

[24] J. Son, T. Hanratty. Numerical solution for the flow around a cylinder at Reynolds numbers of 40, 200 and 500. Journal of Fluid Mechanics 35(2):369-386, 1969. https://doi .org/10.1017/S0022112069001169.

[25] S. Tuann, M. Olson. Numerical studies of the flow around a circular cylinder by a finite element method. Computers and Fluids 6(4):219-240, 1978. https://doi .org/10.1016/0045-7930(78)90015-4.

[26] S. Dennis, G. Chang. Numerical solutions for steady flow past a circular cylinder at Reynolds numbers up to 100. Journal of Fluid Mechanics 42(3):471-489, 1970. https://doi.org/10.1017/S0022112070001428.

[27] C. Christov, R. Marinova, T. Marinov. Does the stationary viscous flow around a circular cylinder exist for large Reynolds numbers? a numerical solution via variational imbedding. Journal of Computational and Applied Mathematics 226(2):205-217, 2009. https://doi.org/10.1016/j.cam.2008.08.022.

[28] H. Lomax, J. Steger. Relaxation methods in fluid mechanics. Annual Review of Fluid Mechanics 7(1):63-88, 1975. https : //doi.org/10.1146/annurev.fl.07.010175.000431 
[29] G. Batchelor. On steady laminar flow with closed streamlines at large Reynolds number. Journal of Fluid Mechanics 1(2):177-190, 1956.

https://doi.org/10.1017/S0022112056000123.
[30] O. Burggraf. Analytical and numerical studies of the structure of steady separated flows. Journal of Fluid Mechanics 24(1):113-151, 1966.

https://doi.org/10.1017/S0022112066000545. 